It is not possible within the limits of an article such as this to do more than touch upon some of the activities of so many-sided a man. Happily there is a good biography of him by H. G. Hutchinson, and "The Life-Work of Lord Avebury" edited by his daughter, the Hon. Mrs. Grant Duff, contains appreciations of his work by leading authorities of the various branches of science which Lord Avebury has enriched by his researches and publications. When reading these we shall gratefully remember how much we owe to this great Victorian naturalist.

F. E. W.

\title{
Stabilisation of Radio Frequencies
}

AMONG the problems which the rapid and A extensive growth of radio communication has presented, is that of keeping the frequencies of all transmitting stations steadily at their assigned values. The success of the various international plans which have been formulated in recent years, particularly for the control of broadcasting, must ultimately depend upon the ability of radio engineers to adjust and maintain a wireless transmitting station at its correct frequency or wave-length. At the present time the primary standards of frequency, which utilise either a tuning fork or a piezo-electric crystal, are amongst the most accurate of our physical standards. With the aid of suitable equipment, there is no difficulty in maintaining and using an accuracy well within one part in a million, while the frequency standards of different countries are in substantial agreement to within a few parts in ten million. Similar types of crystal or tuning fork oscillators can be employed to control the frequency of transmitting stations of appreciable power, by the aid of somewhat elaborate power-amplifying and, if necessary, frequency-multiplying equipment. This arrangement admirably serves the purpose of those stations operating on a single wave-length, and is used with conspicuous success in broadcasting stations and those used for long-distance telegraphic and telephonic communication.

There are, however, many cases, particularly in connexion with ship and aircraft communication, where it is necessary that the transmitting station shall be able to operate on a large number of different wave-lengths and still maintain a high degree of accuracy and stability on each of these wave-lengths. It is usually an accompanying condition of such circumstances that the whole of the transmitting and frequency controlling apparatus must be much simpler than that which is employed at fixed land stations. It is to meet such a demand as this that the Radio Research Board of the Department of Scientific and Industrial Research is at present studying the problem of developing a suitable valve oscillator which will provide frequency stability at a transmitting station without the necessity for elaborate equipment.

As a preliminary to the experimental work, which is now being conducted by the Radio Department of the National Physical Laboratory, a thorough survey of the available information on the subject was made and this has recently been published*. This résumé of the literature has been drawn up in two parts. The first part consists of an essay on the subject as a whole, and comprises in effect a brief textbook of the fundamental principles of this branch of radio science illustrated by reference to typical circuit arrangements used in practice. The second part consists of abstracts of papers representative of the most important published work on the subject, with commentary notes which are intended to bring each particular contribution into perspective with the whole.

In attempting to classify the causes of frequency variations in simple valve-maintained oscillators, a distinction can be drawn between frequency variations due to changes of a purely electrical character and those due to changes of the physical configuration of the system. A simple and admittedly inadequate analysis of the valve-maintained oscillator indicates that frequency variation due to incidental changes in the valve and its circuits can be minimised by meeting certain conditions. Various special circuit arrangements have been developed on these lines and the consequent frequency stabilities obtained are variously estimated at between one and one hundred parts in a million. A more exact analysis shows that it is very difficult to maintain electrical oscillations by means of a valve without producing harmonics which have a detrimental effect upon the steadiness of the fundamental frequency. Experimental data are lacking as to the quantitative significance of this effect, which, however, may be minimised by means of circuits designed to reduce so far as possible the potential differences due to the harmonics generated. Recent investigations have shown that the inter-electrode capacitances of thermionic valves may be expected to vary with the space-charge conditions of the valve, which in turn will vary with supply-voltage and oscillation conditions. Since these inter-electrode capacitances are included in the electrical circuit which determines the frequency of oscillation, any variation in this capacitance will produce a corresponding variation in the frequency.

An ideal valve oscillator is probably one in which the frequency of oscillation is determined solely by the inductance and capacity in the external oscillatory circuit. In this case, however, it is evident that the frequency will be directly

- Department of Scientiflc and Industrial Research. Radio Research, Special Report No. 13: Valve Oscillators of Stable Fre quency : a Critical Survey of Present Knowledge. By'F. M. Colebrook Pp. vii +56. (London: H. M Stationery Office, 1934.) 18. net. 
dependent upon any changes in the physical configuration of this circuit due to changes in atmospheric conditions. For example, changes in temperature of the inductance coils and condensers in the circuit will produce changes in the electrical values of these components to an extent depending upon the coefficients of expansion of the materials used in their construction. The limited experimental evidence so far available indicates that changes of frequency resulting from temperature variations may exceed fifty parts in a million per degree centigrade. To a lesser, but by no means negligible, extent, changes in atmospheric pressure will produce a change in capacit. ance of an air condenser by virtue of the alteration in the dielectric constant of air. A further factor to be taken into account in a complete study of the subject is the effect of the load circuit, which is coupled to the oscillator and by means of which the oscillations generated are put to practical use. A brief consideration of the relevant conditions indicates that in order to minimise the effect of the load circuit on the oscillation frequency, this circuit should be slightly detuned by an amount which depends upon its effective resistance.

In conclusion, the possibility of securing frequency stability in radio transmitting stations by the use of an automatic monitoring arrangement is discussed briefly in the report recently published. The scheme provides for the frequency of the transmitter to be adjusted directly to agree with that of a small power valve oscillator designed for a high-degree of frequency stability under no-load conditions. If such a scheme can be developed successfully without undue complication of equipment, it may provide one solution to the problem of stabilising the frequency of a simple transmitter which has to be operated over a wide range of frequencies. The whole subject is, however, being investigated in a comprehensive manner, since more than one solution may ultimately be necessary to meet the conditions of practical radio communication.

\section{Physiology of the Blue Whale \\ By Prof. August KroGH, Laboratory of Zoophysiology, University of Copenhagen}

\section{RATE OF GROWTH}

T the paper by Mackintosh and Wheeler, 1 "Southern Blue and Fin Whales" ("Discovery Reports", I, 1929), a graph is given showing the growth in length of the Blue whale from the fotal state to maturity. In Laurie's paper, "Some Aspects of Respiration in Blue and Fin Whales" ("Discovery Reports", VII, 1933), graphs are given showing the relation between length and weight of Blue whales, and since this relation is remarkably constant, it becomes possible to calculate the weight of the young whales at different stages.

Such a calculation shows the new-born Blue whale of $7 \mathrm{~m}$. length to weigh about $2,000 \mathrm{kgm}$., while at weaning seven months later, the length has increased to $16 \mathrm{~m}$. and the weight to 23,000 kgm. When the whales become sexually mature at two years of age, the females are on an average $23.7 \mathrm{~m}$. in length and weigh $79,000 \mathrm{kgm}$. The period from weaning to maturity includes two summer seasons (about twelve months) in antarctic waters with abundant food, and one winter in more northern waters where food is scarce. The increase from $23,000 \mathrm{kgm}$. to $79,000 \mathrm{kgm}$. therefore takes place mainly or exclusively during the twelve months when they are in the antarctic. Laurie gives a table showing the composition of a $20 \mathrm{~m}$. Blue whale. Assuming the same composition during the period of growth, the $56,000 \mathrm{kgm}$. increase should be distributed as follows :

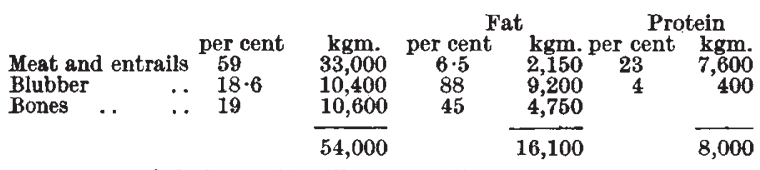

Calories : 150 mill. +44 mill. $=194$ mill. Calories per day in 365 days $=540,000$ " " , , $, 518, "=380,000$
It may be of some interest to compare this rate of growth with that of a pig. The new-born pig weighs about $1 \mathrm{kgm}$. After seven weeks' lactation, the weight is about $6 \mathrm{kgm}$. and, feeding with about $200 \mathrm{kgm}$. of skim milk and $300 \mathrm{kgm}$. of grain, it attains the weight of $90 \mathrm{kgm}$., at which it is converted to bacon at the age of six months. The food consumed corresponds to a little more than 1 million calories, and the increase in weight from weaning to 320,000 calories, or 2,600 cal. per day. Assuming an average weight for the growing pig of $\frac{6+90}{2}=$ about $50 \mathrm{kgm}$. and for the growing whale of $\frac{23,000+79,000}{2}=$ about 50,000 $\mathrm{kgm}$, it is seen that the growth of the pig per unit weight is about five times as rapid as that of the whale. The comparison should not, however, be made on the weight but on the surface basis, corresponding to the cube root of the square of the weight $\left(W^{2 / 3}\right)$. When the whale weighs just 1,000 times the pig, the increase per day should be $1,000^{2 / 3}-100$ times as large, but it is found to be 540,000 calories instead of 260,000 , or just double that of the pig, in spite of the fact that the pig has its food served regularly, while the young whale is left to its own resources. It is a pity that the chemical composition of the crustacean Euphausia superba constituting the whales' staple diet is not known. It would be extremely interesting to know if whale fat is mainly derived directly from the food or mainly built up by synthesis.

\section{Metabolism, Circulation and Respiration}

On the assumption that the surface law is valid for whales and that the normal metabolism is 